\title{
Gedankenverkehr, Kreuzung und verdichtung
}

\author{
Fleck, Simmel und die Völkerpsychologie
}

Rainer Egloff

Communication of Ideas, Intersections and Consolidation - Fleck, Simmel, and Völkerpsychologie

Ludwik Fleck's rediscovery, initiated by Thomas Kuhn, was prominently related to its sociological emphasis. But while this emphasis as such resonated with the sociological circles within science studies, Fleck's actual sociological position was criticized for lacking clear boundaries to psychology and philosophy, and eventually for inconsistency. This article agrees with the former judgement and rejects the latter. It introduces as core and key to Fleck's own sociology the thought style of Völkerpsychologie, and the sociology Georg simmel developed from this tradition.

Keywords: Ludwik Fleck, Moritz Lazarus, Georg Simmel, sociology of science, cultural studies, psychology, folklore

Schlüsse/wörter: Ludwik Fleck, Moritz Lazarus, Georg Simmel, Soziologie der Wissenschaft, Kulturwissenschaften, Psychologie, Volkskunde

Ludwik Fleck wurde bekanntlich dadurch dem Vergessen entrissen, dass Thomas Kuhn 1962 im Vorwort zu seinem epochalen Werk Struktur wissenschaftlicher Revolutionen Flecks damals praktisch unbekannte Monographie Entstehung und Entwicklung einer wissenschaftlichen Tatsache (Basel 1935) als Studie vorstellte, die viele seiner eigenen Gedanken vorweg genommen habe. Kuhn leitete damit nicht nur eine stark verzögerte Erfolgsgeschichte Flecks ein (Kuhn [1962] 1976: 8), er gab dieser posthumen Rezeption auch eine erste Richtung vor. Kuhn hatte im besagten Vorwort die Fleck'sche Monographie nämlich explizit als soziologische Anregung für ihn angesprochen, und infolgedessen fand Fleck erste Aufnahme insbesondere in als soziologisch verstandenen Fragestellungen, Impulsen und Kontexten. ${ }^{1}$ In der bis dahin philosophisch und historisch dominierten Wissenschaftsforschung adoptierte die aufstrebende Wissenschaftssoziologie Fleck als Ahnvater (Cohen/Schnelle 1986: xvi). Als der damals führende amerikanische Wissenschaftssoziologe Robert K. Merton bei der University of Chicago Press, die bereits Kuhns Bestseller im Programm hatte, eine englische Übersetzung 
von Flecks Monographie mitherausgab (Fleck [1935] 1979) und auch bei Suhrkamp eine Neuauflage auf Deutsch erschien (Fleck [1935] 1980), kam die Fleck-Rezeption richtig in Schwung. Dabei zeigte sich ein scheinbares Paradox: Zwar wurde Flecks soziologische Pionierleistung in den höchsten Tönen gelobt, diese ließ sich aber systematisch und historisch nur schwer ins soziologische Feld einordnen. ${ }^{2}$

So fand Fleck kaum Anschluss an die soziologischen Diskurse seiner Zeit, was insbesondere für die während der nationalsozialistischen Herrschaft unterbrochene, marginalisierte oder emigrierte deuschsprachige Wissenssoziologie galt. Thomas Schnelle, der sich wie kein Zweiter um eine vertiefte Rekonstruktion und Rezeption des Fleck'schen Werks verdient gemacht hat und die deutsche Neuausgabe der Monographie (Fleck 1980) sowie ausgewählter Aufsätze Flecks (Fleck 1983) mitherausgegeben hatte, deutete Mitte der 1980er Jahre in einem englischsprachigen Sammelband denn auch die ambivalent gebliebene Rolle Flecks als Soziologe an. Schnelle formulierte vorsichtig, die posthume Rezeption Flecks hätte in erster Linie dessen soziologische Innovationen hervorgehoben, und in diesem Zusammenhang sei wiederholt die Vermutung geäußert worden, Fleck sei mit der damaligen Wissenssoziologie sehr vertraut gewesen, und diese hätte die Entwicklung seines eigenen Ansatzes stark beeinflusst (Schnelle 1986: 6). Bereits zehn Jahre früher hatte William Baldamus im Rahmen einer Festschrift für Norbert Elias das soziologisch innovative Potential von Entstehung und Entwicklung einer wissenschaftlichen Tatsache untersucht. Baldamus wies die Vermutung von Flecks Vertrautheit mit seinerzeit aktueller Soziologie rundweg zurück. Obwohl das Manuskript für die Monographie von Fleck im Sommer 1934 vollendet worden sei, stammten die meisten der zitierten soziologischen Schriften noch aus der ersten Dekade des Jahrhunderts: Jerusalem, Le Bon, Durkheim, Gumplowicz, Lévy-Bruhl, Scheler. Von Max Weber, Georg Lukàcs oder Karl Mannheim habe Fleck nichts gewusst, und er sei gänzlich unvertraut gewesen mit der rasanten Entwicklung der empirischen Soziologie in den USA der frühen 1930er Jahre. All dies erkläre, warum Flecks soziologischer Stil oft seltsam altmodisch klinge. So hätte Fleck von den frühen Soziologen etwa den Mangel an klaren Grenzen zwischen Soziologie, Psychologie und Sozialphilosophie übernommen (Baldamus 1977: 140). Baldamus hatte als einer der ersten bereits in den 1960er Jahren versucht, Flecks Beitrag für die damalige Soziologie zu rekonstruieren und fruchtbar zu machen. Seine Stoßrichtung zielte auf den Aufbau und die Abgrenzung der Soziologie als Fach. In den 1970er Jahren hatte die Soziologie in Europa - und mit über einem halben Jahrhundert Verspätung gegenüber den USA - gerade den Durchbruch als eigenständige akademische Fachdisziplin geschafft und stand im Begriff, ihren Status weiter zu verbessern. ${ }^{3}$ Sowohl durch die Identifikation von genuinen Pionieren und Klassikern als auch den Aufbau eines unverwechselbaren methodischen und theoretischen Profils, das sich an den 
naturwissenschaftlichen Forschungsidealen ausrichtete, suchte die Soziologie Legitimation und Unabhängigkeit gegenüber ihren Nachbar- beziehungsweise Ursprungsfächern wie der Philosophie oder der Geschichte. Aus dieser Perspektive war Baldamus' kritische Beurteilung von Fleck zweifellos zutreffend.

Fleck hat die Versuche der Rezeption, ihn systematisch oder historisch an bedeutende soziologische Diskurse anzuschliessen, noch dadurch erschwert, dass er selbst für seinen Ansatz zwar immer wieder "Soziologie” reklamierte, sich jedoch zu fast allen von ihm als soziologisch genannten Autoren kritischablehnend verhielt, da jene naturwissenschaftlichem Wissen zu respektvoll begegneten. ${ }^{4}$ Fleck schien also seine soziologische Emphase nicht an disziplinär legitimierte Diskurse rückbinden zu können oder zu wollen. Dies könnte als ein plausibler Grund dafür genannt werden, dass Fleck im soziologischen Feld zu Lebzeiten tatsächlich kaum Beachtung gefunden hat. Da nun aber Thomas Kuhn und andere Berühmtheiten Fleck gerade wegen seines soziologischen Ansatzes für wichtig erklärten, musste es ebenso plausibel erscheinen, Flecks Soziologie als in der Disziplingeschichte bis dato zu Unrecht ignorierten Solitär zu verstehen und einer Wissenschaftssoziologie der Gegenwart als systematisch klassischen Bestand anzutragen. In einer Sammelbesprechung von Fleck im Kontext einer Soziologie des Wissens kam Jonathan Harwood 1986 jedoch zu einem ernüchternden Urteil: Flecks Hauptwerk enthalte mit der konzeptionellen Betonung der kollektiven Natur wissenschaftlicher Entdeckung zwar einen innovativen soziologischen Kern, dieser sei jedoch wegen seiner abstrakten, unsystematischen, wenig trennscharfen und inkonsistenten Ausführung überholt und allein von historischem Interesse (Harwood 1986). Harwood fügte hinzu, wenn Flecks Werk für die künftige Soziologie des Wissens von Bedeutung sein wolle, müssten dessen Verehrer ihre Erfindungsgabe bemühen, um die bei Fleck fragmentarischen Konzepte zu einer kompletten Theorie umzugestalten. Ein erfolgreiches Resultat wäre allerdings eine kollektive Errungenschaft, die in ihren Grundzügen nur eine schwache Verbindung zu den ursprünglichen Intentionen Flecks aufwiese (Harwood 1986: 186).

Dass die Fleck'sche Soziologie gewisse Lücken aufweist, soll hier nicht bestritten werden, und auch nicht, dass Fleck als Sozialwissenschaftler ein Amateur war (Baldamus 1977: 140). Nichtsdestotrotz kann Fleck - so die hier vertretene These - einen wichtigen, fruchtbaren und konsistenten Beitrag zur Wissenschaftssoziologie und allgemein zu einer Wissenschaft des Sozialen leisten. Diesen Beitrag adäquat zu erschließen fällt aus dem soziologischen Mainstream der zweiten Hälfte des 20. Jahrhunderts heraus tatsächlich schwer. Besser lässt sich Flecks Ansatz einerseits im Laufe der Fachgeschichte verdrängten protosoziologischen Denkstilen des späten 19. und frühen 20. Jahrhunderts, andererseits aber auch aktuellen - sozusagen postdisziplinären - Strömungen des 21. Jahrhunderts anschließen. Nachfolgend möchte ich Harwood also mehrfach widersprechen. Zum Einen will ich mögliche 
rekonstruktive Wege andeuten und Hinweis geben, wie Fleck als Soziologe konsistenter, präziser und somit in seinen Stärken neu gelesen werden kann, ohne dass dies von Fleck selbst wegführen muss. Solche Lesart muss nicht zwingend auf eine komplette Theorie im Sinne Harwoods ausgerichtet sein. Sie darf sich überdies den disziplinstrategischen Emphasen aus Harwoods Zeit, wo etwa detaillierte Sozialstrukturanalyse oder die systemtheoretische Differenzierung von (wissenschaftlichen) Denkstilen im Vordergrund standen, auch verweigern. Ansatzweise soll vielmehr einer Fleck'schen Soziologie nachgegangen werden, wie sie im Rahmen heutiger interdisziplinärer Öffnung aufbauend wirken kann. Dabei soll insbesondere die spezifische Rolle beleuchtet werden, die soziologische Aspekte bei Fleck im Rahmen der gesamten Theorie spielen, und wie diese mit den Funktionen von Nachbarfeldern wie Geschichte, Ethnologie beziehungsweise Volkskunde sowie Psychologie und Philosophie zusammenhängen. ${ }^{5}$ Denn in einer Verortung von Flecks Wissenschaft des Sozialen müssen diese Interaktionen einen wichtigen Stellenwert einnehmen, und in solcher Kontextualisierung erscheint Flecks Ansatz auch konsistenter als unter dem Licht des soziologischen Theorienregimes der 1970er und 1980er Jahre.

Werkgeschichtlich fokussiert auf Flecks Monographie von 1935 möchte ich diese Beziehungsgeschichte insbesondere anhand der Stichworte Verdichtung, Kreuzung und Verkehr diskutieren. Als verbindende Spuren zwischen den Fächern, Denkstilen und Autoren bei Fleck können zunächst explizite Fußnotenreferenzen und Namensnennung dienen, dann übernommene oder zumindest ähnliche Begrifflichkeiten und Konzepte. Schließlich werde ich mutmaßlichen Verwandtschaften der Fleck'schen Quelle auch etwas weniger eng am Text nachspüren, um auf systematische Berührungen, Überschneidungen sowie mögliche Ergänzungen hinzuweisen. Als systematisch verwandten Ansatz und impliziten Bezug Flecks stelle ich die Völkerpsychologie von Lazarus und Steinthal vor, und als explizite und positive Referenz möchte ich den Lazarus-Schüler Georg Simmel in den Vordergrund rücken. Die beiden Bezugsgrößen sind genealogisch eng miteinander verbunden und können als eine Traditionslinie verstanden werden. Diese hat in der Fleck-Rezeption bislang kaum Beachtung gefunden, enthält aber einiges Potential als missing link. Auffällig ist, dass die Völkerpsychologie und die Soziologie Simmels genauso wie die Fleck'sche Programmatik die Fachgrenzen sprengen. Sie verhalten sich dem modernen Fächerkanon gegenüber genuin transdisziplinär - um nicht zu sagen undiszipliniert. Gerade deshalb wurden sie im Rahmen sozialwissenschaftlicher Akademisierung, Kompartmentalisierung, Ein- und Abgrenzung zeitweilig marginalisiert und weitgehend vergessen. ${ }^{6}$ Im Zuge jüngerer Entwicklungen zur Interdisziplinarität und zur Erosion traditioneller Fachidentitäten, der mit einer neuerlichen kulturalistischen Wende in den Sozialwissenschaften einhergeht, erscheinen diese Projekte heute jedoch wieder verblüffend frisch. 


\section{Zur Selbstkontextualisierung Flecks im Rahmen einer Wissenschaft des Sozialen}

Ausgehend von der erwähnten Rezeption des Fleck'schen Werks als zunächst vor allem soziologisches möchte ich auf einige Facetten von Flecks Programm hinweisen, die dieses im genannten Beziehungsgeflecht disziplinärer Denkstile besser positionieren lassen und es soziologischer Engführung entziehen.

Wenn wir uns dem Anspruch von Entstehung und Entwicklung einer wissenschaftlichen Tatsache über Titel und Inhaltsverzeichnis nähern, lässt sich dieser analytisch stark kondensiert erfassen. Bereits der Haupttitel des Werks proklamiert Entscheidendes: Die Gewordenheit und aktive Prozessualität jeder wissenschaftlichen Tatsache verweist auf deren historische Kontingenz und auf das Doppelprogramm einer historischen Epistemologie. ${ }^{7}$ Philosophisch-erkenntnistheoretisch vertritt dieses Programm eine relativistische beziehungsweise relationistische Position, ${ }^{8}$ es verfährt jedoch auch argumentativ-methodisch historisch, indem es empirisch-analytisch die Geschichte einer bestimmten medizinisch-wissenschaftlichen Tatsache nachzeichnet - als, wie Fleck sagt, „Ereignis der Denkgeschichte und als denkkollektives Widerstandsaviso“ (Fleck [1935] 1980: 111). Besonders provokativ am Titel ist das nachgestellte Wort „Entwicklung“, denn ist die wissenschaftliche Tatsache einmal entstanden, festgestellt oder entdeckt - so könnte man meinen - steht sie doch fest und muss beziehungsweise kann sich nicht mehr entwickeln, wird höchstens ersetzt. Solche Ansicht herauszufordern, ist Flecks Ziel: für ihn sind sowohl Denken als auch Tatsachen veränderlich (Fleck [1935] 1980: 69). Flecks Historismus ist evolutionistisch, jedoch nicht im Sinne eines Nachvollzugs von Entwicklung zum Höheren, Rationaleren oder Besseren, sondern als Ausdifferenzierung, als Entfaltung und Reduktion von Unterschieden, als Dynamik von Komplexität. Wenn es bei Fleck so etwas wie teleologische Vollendung gibt, dann bleibt diese quasi lokal, singulär, spezifisch. Diese Anschauungen sind aus Flecks Ausbildung und Praxis als Mediziner und Mikrobiologe abgeleitet. So vergleicht er die Entwicklung von Denkstilen etwa mit der Ontogenese eines Organismus oder dem zeitlichen Verlauf einer Krankheit:

Mich lehrte die Biologie, ein der Entwicklung unterworfenes Gebiet immer entwicklungsgeschichtlich $\mathrm{zu}$ untersuchen. Wer betriebe heute Anatomie ohne Embriologie? Genauso bleibt jede Erkenntnistheorie ohne geschichtliche und vergleichende Untersuchungen ein leeres Wortspiel, eine Epistemologia imaginiabilis. (Fleck [1935] 1980: 31)

Gehen wir vom Titel zum Inhaltsverzeichnis über, tritt die begriffsgeschichtliche Betonung in Flecks Ansatz deutlich hervor, die mit dem erkenntnistheoretischen Interesse verbunden ist. Fleck betreibt seine Begriffsgeschichte mehrdimensional: als Nachvollzug von Bedeutungsentwicklung 
(quasi als semantische Geschichte) und von „Ideenentwicklung“ (Fleck [1935] 1980: 39), aber auch als Geschichte von begriffsverbundenen Praktiken, von Mythologie, Sitte und Brauch. Damit lässt sich Fleck, der auch explizit „,kulturhistorisch“ argumentiert (Fleck [1935] 1980: 16), mit einer auf Hegel, ${ }^{9}$ Herder und andere zurückgehende Theorie individueller und kollektiver Geist-Entwicklung, sowie den Traditionen von Volkskunde einerseits und von Völkerkunde andrerseits in Verbindung bringen. ${ }^{10}$ Ich möchte hier besonders auf Affinitäten von Flecks Programm zu jenem der Völkerpsychologie von Lazarus und Steinthal hinweisen.

Zwischen den 1850er und 1890er Jahren und insbesondere ab $1861 \mathrm{mit}$ ihrer Zeitschrift für Völkerpsychologie und Sprachwissenschaft propagierten und beschrieben Moritz Lazarus und Heymann Steinthal ein sich von Hegel absetzendes und eng an Wilhelm von Humboldts Sprachwissenschaft angelehntes Programm, das bereits von Durkheim als eigentlich „sozialpsychologisch“ identifiziert wurde (Köhnke 2003: IX). Wohl nahmen sich Lazarus und Steinthal den „Volks-“ oder „Nationalgeist“ beziehungsweise die "Volksgeister“ zum Gegenstand und suchten die ,innere geistige oder ideale Thätigkeit eines Volkes - in Leben, Kunst und Wissenschaft“ sowie „,die Gründe, Ursachen und Veranlassungen sowohl der Entstehung als der Entwicklung und letztlich des Unterganges der Eigenthümlichkeiten eines Volkes zu enthüllen" (Lazarus [1851] 2003: 4, Hervorhebung im Original). Nicht zufällig aber verorteten Lazarus und Steinthal ihr Programm systematisch in der Psychologie, und mit ihrer Rückbindung an das "Innerste des Geistes“ grenzte sich diese Völkerpsychologie von einer physiologisch orientierten Anthropologie ebenso ab wie von der Ethnologie. Letztere wurde von Lazarus und Steinthal der Zoologie zugeschlagen, da sie den Menschen als Tier und Naturereignis, in seiner leiblichen Lebensweise betrachte (Böhme/Matussek/ Müller 2002: 37). Schließlich interessierte sich die Völkerpsychologie nicht nur für ganze Völker und Nationen, sondern für Gruppen aller Art. Sie widmete sich allgemein dem „Verhältniß des Einzelnen zur Gesammtheit“ (Lazarus [1883/1862] 2003). Dieses Verhältnis wurde von Lazarus als interaktiv betrachtet und als „Wechselwirkung“ konzeptualisiert, denn gemäss Lazarus ist das Individuum ohne jede Gruppenzugehörigkeit undenkbar und umgekehrt sind Gruppen stets aus Individuen zusammengesetzt (Klautke 2010: 6).

Fleck bezieht sich meines Wissens nirgends explizit auf die Völkerpsychologie. Konzeptuelle Parallelen sind jedoch offensichtlich, nicht zuletzt im Fokus auf die Denk- oder Geistestätigkeit von Kollektiven sowie im historischen beziehungsweise prozessualen und insbesondere begriffsgeschichtlichen Zugang, der die Differenzierung gemäß einzelnen Gruppen sowie das Spiel zwischen diesen stärker betont als die Aufhebung in einem übergeordneten System „Volk“ oder „Gesellschaft" oder „Wissenschaft“" ${ }^{11}$ 


\section{Verdichtung}

Mit dem Begriff der „Verdichtung“ nimmt Fleck einen der zentralsten völkerpsychologischen Begriffe überhaupt auf. In Lazarus' Schrift zur „Verdichtung des Denkens in der Geschichte“ von 1862 wird Verdichtung als Kondensations-, Vereinfachungs- und Konzentrationsmodus eingeführt, der zur Vermittlung von kulturspezifischen Inhalten, Vorstellungen, Denk- und Handelsweisen dient und damit zum Identität stiftenden Kulturmedium par excellence wird (Lazarus [1862] 2003, Graevenitz 1999). Verdichtung vollzieht sich

in der Geschichte für einzelne Völker und selbst für die die gesammte Menschheit; sie geschieht, indem Begriffe und Begriffsreihen, welche in früheren Zeiten von den begabtesten Geistern entdeckt, von wenigen kaum erfaßt und verstanden, doch allmächlich zum ganz gewöhnlichen Gemeingut ganzer Classen, ja der gesammten Masse des Volkes werden können. (Lazarus [1862] 2003: 27)

Der „Prozeß der Verdichtung der Begriffsmassen“ erlaubt sowohl die „fortschreitende Ausbreitung der Ideen“ als auch „die immer allgemeinere Vertiefung in der Anschauung der Dinge“ (ebd.: 29). Verbreitung und Vertiefung werden im gleichen Zug ermöglicht, weil Eingewöhnung und Geläufigkeit die verdichteten Ideen letztlich als einfach erscheinen lassen. Die Ideen sind dann leicht zu erfassen, zu erlernen und zu vermitteln, und weil sie als klar und solide erscheinen bieten sie Hand $\mathrm{zu}$ ihrer weiteren Differenzierung:

Alle Bildung des Einzelnen - also auch der Gesammtheit - gründet sich auf Aneignung und Verarbeitung beziehungsweise [sic] einfacher Begriffe; an die elementaren Denkformen und Anschauungsweisen, in denen die Welt und das Leben erfaßt wird, knüpft die Entwicklung des Geistes an. Das Maß der Klarheit eines Gedankens steht mit dem Maße der Anstrengung, ihn zu erfassen, im umgekehrten Verhältniß; was aber ohne Anstrengung erfaßt werden kann, gilt als das Einfache, Elementare, womit der Pädagoge seine Bildungsarbeit beginnt. Je mehr also eine Begriffsreihe, jetzt noch das Product einer kaum geahnten Entdeckung und Gegenstand einer mühevollen Erkenntnisarbeit, bei der einen Generation an Klarheit und Durchsichtigkeit und Geläufigkeit gewinnt, desto mehr erhält sie von jener elementaren Natur und kann für eine folgende Generation Gegenstand der ersten und einfachsten Belehrung sein. (Ebd.)

In Lazarus' völkerpsychologischen Konzeptualisierung scheint eine bemerkenswerte Nähe zu Flecks Ansatz und Begrifflichkeit auf, wo vorwissenschaftliche „Urideen (Präideen)“ (Fleck [1935] 1980: 35), exoterisches Wissen und Popularisierung dem esoterischen wissenschaftlichen Fachwissen dialektisch beziehungsweise interaktiv gegenüber stehen:

Gewißheit, Einfachheit, Anschaulichkeit entstehen erst im populären Wissen; den Glauben an sie als Ideal des Wissens holt sich der Fachmann von dort. Darin liegt die allgemeine erkenntnistheoretische Bedeutung populärer Wissenschaft. (Ebd: 152) 
Gemäss Fleck sind Eingeweihten oder gar mit bestimmten Ideen Aufgewachsenen diese Ideen ganz und gar selbstverständlich. Gewisse Wissenselemente sind nur in einem bestimmten Denkkollektiv lebendig und verstanden, andere sind althergebrachtes Allgemeingut - „Volkswissen“ (ebd.: 33). Sowohl bei Lazarus wie bei Fleck gibt es einen kontinuierlichen Übergang zwischen Wissenschaft und anderen Alltagspraktiken. Herauszustreichen ist sodann die gemeinsame besondere Aufmerksamkeit für Sprache, welch letztere ihre wichtige Rolle doch stets auch mit Verdichtungen bildnerischer (ideographischer) und praktischer Art teilen muss. Lazarus schreibt dazu:

Außer der Sprache aber sind es die Sitten und die sittlichen Institutionen aller Art, welche dem Menschen den sittlichen Gehalt seiner Zeit, das Product langer geistiger Entwicklung und historischer Arbeit eben so fertig entgegenbringen, wie die vorhandenen Kunstwerke, die Monumente des Genies und des Fleißes, ihm die allmählich gereifte ästhetische Anschauung göttlicher und menschlicher Dinge vor die Seele führen, um sein Gemüth, wenn es empfänglich ist, auf die gleiche Höhe zu erheben, und es mit der gleichen Idealität zu erfüllen. (Lazarus [1862] 2003: 32)

Thesenhaft möchte ich die Lazarus'sche Verdichtung als verwandt, affin oder wenigstens kompatibel mit Flecks Konzeptionalisierung von „Anschauungen als selbstständige[n] stilvolle[n] Gebilde[n] “ (Fleck [1935] 1980: 40), der „Beharrungstendenz der Meinungssysteme“ (ebd.: 53) und dem Zentralbegriff des Denkstils konstatieren. Der moderne wissenschaftliche Denkstil ist an eine Denkgemeinschaft gebundene, in theoretischen Sätzen, Sinnbildern oder Ideogrammen, rituellen Praktiken und Lehrveranstaltungen, Apparaten, Stimmungen, Erfahrungen und Idealen stilvoll verdichtete Anschauung.

Fleck übernimmt den Verdichtungsbegriff allerdings nicht direkt von Lazarus. Er verweist dafür auf Wilhelm Jerusalem, welcher den Begriff seinerseits modifiziert als "soziale Verdichtung“ im Ursprung sich selbst zugeschrieben hatte (ebd.: 53, 64, 65, 67). ${ }^{12}$ Bei der sozialen Verdichtung Jerusalems steht ein „Prozess der gegenseitigen Verstärkung“ im Vordergrund, wie er sich ,,keineswegs bloss bei den Primitiven“ sondern „,auch heute noch im täglichen Leben“ und „selbst in der Wissenschaft" finde (ebd.: 64 f.). Fleck führt den Begriff „,soziale Verdichtung“ im Kontext seiner Auseinandersetzung mit soziologischer Literatur auf und analogisiert ihn auch mit „sozialer Verstärkung":

Eine einmal veröffentlichte Aussage gehört jedenfalls zu den sozialen Mächten, die Begriffe bilden und Denkgewöhnungen schaffen: sie bestimmt gemeinsam mit allen anderen Aussagen, was man ,anders nicht denken kann." Auch wenn sie bekämpft wird, wächst man mit ihrer Problematik auf, die innerhalb der Gesellschaft kreisend, zur sozialen Verstärkung gelangt. [„Verdichtung“ wie Jerusalem sie nennt.] Sie wird selbstverständliche Realität, die dann ihrerseits weitere Erkenntnisakte bedingt. Es entsteht so ein geschlossenes, harmonisches System, innerhalb dessen der logische Ursprung einzelner Elemente nicht mehr aufzufinden ist. (Ebd: $52 \mathrm{f}$.) 
Das Konzept der Verdichtung wird so bei Fleck zu einem argumentativen Schlüssel zur Begründung seines Standpunkts einer „soziale[n] Bedingtheit jedes Erkennens" (ebd.: 53).

Doch kaum hat Fleck in diesem Zusammenhang die „Bedeutung soziologischer Methode für die Untersuchung geistiger Betätigung“ als schon seit Comte bekanntes und neuerlich von der Durkheimschule und Jerusalem betontes Phänomen konstatiert (ebd.: 62) und Lévy-Bruhls und Jerusalems Anliegen skizziert, übt er vehemente Kritik: ${ }^{13}$

Nun begehen alle diese soziologisch und humanistisch gebildeten Denker - so fördernd ihre Gedanken sind - einen charakteristischen Fehler: sie haben allzugroßen Respekt, eine Art religiöser Hochachtung vor naturwissenschaftlichen Tatsachen. (Ebd.: 65)

Flecks Kritik gilt der Vorstellung, naturwissenschaftliches Denken entstehe entwicklungssoziologisch dank der Herauslösung der Individuen aus ihrer rigiden Einbindung in mystische Kollektivvorstellungen. Die Individuen zeigten sich dadurch zunehmend empfänglich für „objektive Eigenschaften“ und ,eigentliche Wahrnehmung“, die „Geistesart der Gesellschaften“ würden „für die Erfahrungen zugänglich“ und „gleichzeitig für den Widerspruch empfindlicher" (ebd.: 65 f.). Demgegenüber beharrt Fleck darauf, niemand könne von sich aus ein Gefühl oder eine Kenntnis haben, was physisch möglich oder unmöglich sei, es existiere „keine „Erfahrung an sich“, der man zugänglich oder unzugänglich sein könne" (ebd.: 66). Fleck attackiert Jerusalem für dessen Glaube an gefühlsfreies, objektives und rein theoretisches Denken, das dem zur „selbständigen und eigenkräftigen Persönlichkeit [...] erstarkten Einzelmensch" im Laufe der gesellschaftlichen Entwicklung möglich werde (ebd.: 66 f.). Diese von Jerusalem „Zusammenhang von Tatsache und Individuum" genannte These sei unvereinbar mit Jerusalems eigenem Konzept sozialer Verdichtung (ebd.: 67). Für Fleck ist Erfahrung immer selbst im Bereich eines einzelnen, abgegrenzten und überschaubaren wissenschaftlichen Experiments - ,bereits als verwickelte Erzogenheit zu verstehen, die auf Wechselwirkung zwischen dem Erkennenden, dem bereits Erkannten und dem Zu-Erkennenden beruht“ (ebd.: 17). „Ideenentwicklung“ (ebd.: 39) vollzieht sich dabei nicht als Abstraktion vom Einzelnen zum Allgemeinen, sondern als Differenzierung beziehungsweise Spezialisierung vom Allgemeinen zum Besonderen (ebd.). Soziale Verdichtung und Differenzierung werden bei Fleck zu Zentralkonzepten. Nicht zunehmendes Abstraktionsvermögen des Individuums, sondern kollektive Spezialisierung am Besonderen ist bei Fleck jedoch deren Folge und Funktion.

Wenigstens dreierlei ist in diesem Zusammenhang bemerkenswert: Erstens argumentiert Fleck selbst quasi völkerpsychologisch um die als soziologisch deklarierten Positionen Jerusalems und Lévy-Bruhls zu attackieren. Zweitens beansprucht Fleck für sein eigenes Programm - und damit bin ich wieder bei der Kondensierung des Fleck'schen Programms - auch das 
Etikett der Soziologie. Drittens stellt sich die Frage, welche spezifische Aufgabe Fleck für diese Soziologie vorsieht. Es ist zunächst die allgemeine Betonung der sozialen Dimension jedes Erkennens, welche neben die psychologische und die historische tritt. Dann scheint Fleck der "Soziologie des Denkens“ aber insbesondere auch die Analyse der Formen oben genannter gesellschaftlicher Verdichtung und Differenzierung zuzuweisen. Solche Analysen der „formale[n] Seite wissenschaftlichen Betriebs" (ebd.: 57) liefern zusammen mit den Inhalten den Gegenstand der vergleichenden „Lehre vom Denkstil und Denkkollektiv“. Jede wissenschaftliche Arbeit ist für Fleck kollektiv (ebd.), je nach sozialer Struktur, Größe, Organisation, Hierarchisierung usw. prägt jedoch die „formale Seite wissenschaftlichen Betriebes“ deren Inhalt unterschiedlich (ebd.: 57 f.). Da Fleck in seiner vergleichenden Erkenntnistheorie keinen strengen kategorialen Unterschied zwischen wissenschaftlichen und anderen „Denkgemeinschaften“ macht, kann er etwa das „Mode-Denkkollektiv“ mit seiner Struktur und „Stimmung ebensowie „das Mode-Denken als solches" zum Vergleich heranziehen (ebd.: $141 \mathrm{f}$.).

Mit seiner quasi-völkerpsychologischen Soziologie gesellschaftlicher Formen tritt Fleck in die Fußstapfen des Lazarus-Schülers Georg Simmel. Wie Fleck operierte dieser dezidiert interdisziplinär zwischen Philosophie, Ethnologie, Psychologie, Geschichte und Soziologie. Simmels 1882 in Lazarus' und Steinthals Zeitschrift für Völkerpsychologie und Sprachwissenschaft erschienene Dissertation bringt dies schon im Titel zum Ausdruck. Sie ist „psychologischen und ethnologischen Studien über Musik“ gewidmet. Simmel hat das völkerpsychologische Projekt weitergetragen und um die Jahrhundertwende vor allem auch in die Soziologie als Wissenschaft gesellschaftlicher Differenzierung und gesellschaftlicher Formen überführt (Köhnke 1996, Tyrell/Rammstedt/Meyer 2011, Nedelmann 1995). Bemerkenswerterweise ist Simmel der einzige von Fleck als Soziologen angesprochene Autor, von dem sich dieser nicht auch kritisch distanziert (Fleck [1935] 1980: 145).

\section{Verkehr und Kreuzung}

Fleck bezieht sich in Entstehung und Entwicklung einer wissenschaftlichen Tatsache nur ein einziges Mal auf Simmel, und dies lediglich knapp in einer Fußnote (ebd., Fußnote. 7). Dennoch ist - so meine These - der SimmelBezug für ein Verständnis von Fleck entscheidend. ${ }^{14}$

Die oben erwähnten „Wechselwirkung“ von Lazarus, findet sich als „gedankliche Wechselwirkung“ bei Fleck wieder. Zunächst taucht sie zentral in der Beschreibung eines der wichtigsten und originären Begriffe Flecks auf jener des Denkkollektivs. Dessen Definition als „Gemeinschaft der Menschen, die im Gedankenaustausch oder in gedanklicher Wechselwirkung stehen" 
(ebd.: 54) sowie als ,immer dann vorhanden, wenn zwei oder mehrere Menschen Gedanken austauschen" (ebd.: 60) gemahnt wohl nicht zufällig an den Wortlaut der von Simmel bewusst weit ausgelegten Definition von Gesellschaft überhaupt, die „,da existiert, wo mehrere Individuen in Wechselwirkung treten“ (Simmel [1908] 1992: 17). Im Modus des „Denkverkehrs“ (Fleck [1935] 1980: 1939) bildet die gedankliche Wechselwirkung bei Fleck die Grundlage sowohl von intrakollektiver Denkstilbestärkung wie von interkollektiver Denkstilveränderung. Zusammen mit „,fortwährende[r] Bewegung“ betreibt sie „das Getriebe der Ideen und Wahrheiten“, welches Fleck als antifundamentalistisches Wissensideal hochhält (ebd.: 70). Bei der Erörterung solchen „Gedankenverkehrs“ (Fleck [1935] 1980: 145) verweist nun Fleck auf Georg Simmel: „Den Soziologen ist die Wirkung des sozialen Verkehrs der Denkgebilde grundsätzlich bekannt" schreibt Fleck mit Blick und Fußnotenreferenz auf Simmels Soziologie von 1908 (ebd.: 145 und Fußnote 7). Simmel wird für Fleck somit zum wichtigsten Vermittler sowohl einer positiven Identifikation mit Soziologie wie des völkerpsychologisch Denkstils. Auch die einfachste Wissensvermittlung ist nie ohne Transformation zu haben. Im Modus der Verdichtung führt der „Gedankenverkehr“ oder „Denkverkehr“ innerhalb von Denkkollektiven zur Disziplinierung und Beharrung des jeweiligen Denkstils. Umgekehrt verschieben oder verändern sich bei der interkollektiven Gedankenwanderung die „Denkwerte [...] in einer ganzen Skala der Möglichkeiten [...]: vom kleinen Färbungswechsel über fast vollständigen Sinnwechsel bis zur Vernichtung jedes Sinnes" (ebd.: 143 f.).

Es fällt auf, dass Fleck seiner Fußnotenreferenz auf Simmels Soziologie im Zusammenhang mit den Phänomenen des Denkverkehrs einen präzisierenden Hinweis auf „Kapitel II, worin er die soziale Differenzierung bespricht“ hinzufügt (ebd. 145, Fußnote 7). ${ }^{15}$ Im zweiten Kapitel seiner Soziologie („Die quantitative Bestimmtheit der Gruppe") bespricht Simmel tatsächlich die soziologische Relevanz quantitativer Differenzierung von Gruppen (Simmel [1908] 1992: 63-159]. Als Vertreter und Fortführer der Völkerpsychologie und mehr als jeder andere Soziologe hat sich Simmel Gruppen aller Größen, besonders aber kleinen Gruppen gewidmet. Das Fleck'sche Denkkollektiv, das seinen Ausgang mehr oder weniger spontan in einem ,zweipersonalen Kollektiv“ nimmt (Fleck [1935] 1980: 60), entsteht als „Zweiergruppe“ bei Simmel etwa durch das Teilen eines Geheimnisses (Simmel [1908] 1992: 101). Bei Fleck wie bei Simmel kann sich der Gruppenumfang im Laufe der Zeit vergrößern und stabilisieren, aber auch wieder zerfallen - je größer und je beständiger jedoch das Kollektiv, desto mehr tritt die Relevanz des einzelnen Individuums für die Gruppe zurück. Auch in weiteren Belangen fügt sich die Fleck'sche Konzeption von Denkkollektiven besser in die Simmel'schen Analysen als in andere klassische soziologische Systeme ein. Fleck wie Simmel fokussieren vehement auf die Prozessualität (Nedelmann 1995), die Historizitiät und die Formen des Sozialen. Sie interessieren sich besonders für 
Mechanismen der Selbsterhaltung beziehungsweise Reproduktion von Gruppen, für ihre Strukturiertheit etwa in Hierarchien, Lehrer-Schüler-Verhältnissen, Eliten und Massen, für Konflikte und Konkurrenz, aber auch für Freundschaft (Fleck [1935] 1980: 58 f.). Simmel ist von beiden Autoren soziologisch der Ausführlichere, Genauere, Systematischere, Konsistentere. Daraus kann eine Rekonstruktion des Fleck'schen Ansatzes zweifellos gewinnen. Bei genauerem Hinsehen erweist sich aber schon Flecks Analyse sozialer Strukturen als präziser, als dies Harwood in seiner Kritik gelten lassen wollte. Wenn Fleck die ,spezielle Struktur des wissenschaftlichen Denkkollektivs" (Fleck [1935] 1980: 146 f.) bespricht, ist sein Interesse jedoch in einer Art fokussiert, die sich nicht in den soziologischen Mainstream der 1980er Jahre fügt, wo noch systemtheoretische und strukturfunktionalistische Makrotheorien dominieren. Fleck interessiert sich insbesondere dafür, „wie sich das Esoterische und Exoterische im Rahmen der Wissenschaft auswirkt" (ebd.) - im Universum von Völkerpsychologie und Simmel eine durchaus naheliegende Frage. Das Verhältnis von Fachleuten und Laien und ihrem Wissen, von esoterischem Zentrum und exoterischer Peripherie (ebd.: 155) und die verschiedenen publizistischen Wissenschaftsformen von Zeitschriftbis Lehrbuchwissenschaft (ebd.: 148) werden in den Blick genommen. Wissenschaftliche Arbeitsteilung wird ebenso differenziert wie unterschiedliche Motive die aus dem „soziale[n] Gepräge des wissenschaftlichen Betriebes“ inhaltlich folgen: „Propaganda, Nachahmung, Autorität, Konkurrenz, Solidarität, Feindschaft und Freundschaft" (ebd.: 59). Dabei gehören Inhalte und Formen der Wissenschaft stets wesentlich zusammen. Fleck konstatiert:

Betrachtet man zunächst die formale Seite wissenschaftlichen Betriebs, so ist dessen soziale Struktur unverkennbar. Wir sehen eine organisierte Kollektivarbeit mit Arbeitsteilung, Mitarbeit, Vorbereitungsarbeit, technischer Hilfe, gegenseitigem Ideenaustausch, Polemik etc. (Ebd.: 57 f.)

Während ein Denkstil im intrakollektiven Denkverkehr zunehmend rigide und konservativ wird, kommt Veränderung durch interkollektiven Gedankenaustausch zustande. Im Rahmen von Flecks Betonung denkerischer Kollektivität vielleicht unerwartet, wird hier dem Individuum eine zentrale Rolle zugewiesen. Auch hier lassen sich bei Fleck die Simmel'schen und letztlich völkerpsychologischen Einflüsse geltend machen. Wie Gerhart von Graevenitz in seinem Essay zur „Verdichtung" als völkerpsychologischem Kulturmodell festhält, kam über Simmels frühe Schrift „Über soziale Differenzierung" (1890) die völkerpsychologisch formulierte, auf Herbart zurückgehende Konstruktion des Individuums als „Kreuzungspunkt unzähliger sozialer Fäden“ in die Soziologie (Graevenitz 1999: 35). Von Simmel wiederum übernimmt Fleck diese Figur vom Individuums als Schnittpunkt sozialer Kreise (Köhnke 1996: 321-333) und implementiert sie zentral in seine 
Theorie. Im Individuum verschränken sich verschiedene Denkkollektive und stile:

Ein Denkkollektiv besteht aus vielen [...] sich überkreuzenden Kreisen, ein Individuum gehört mehreren exoterischen Kreisen und wenigen, eventuell keinem esoterischen an. (Fleck [1935] 1980: 138)

Das Individuum wird so zur Kreuzung zwischen Denkstilen und Denkkollektiven. Über das Individuum vollzieht sich der interkollektive Denkverkehr und damit auch potentielle Innovation. Das Individuum tritt also bei Fleck als Drittes im Erkenntnisprozess zwischen Kollektiv und Objekt. Dieses Individuum ist aber nicht ein aus dem Sozialen herausgelöstes, dem Gesellschaftlichen Entgegengesetztes. Es ist vielmehr ein in gesellschaftlichen Verschränkung wachsendes, potenziert Soziales: Individualisierung erfolgt gerade durch Teilhabe an mehreren Kollektiven.

\section{Flecks Soziologie des Denkens}

Ausgehend von der post-Kuhn'schen Fleck-Rezeption, die zunächst soziologisch dominiert war und die soziologischen Gehalte bei Fleck hervorhob, habe ich Flecks Selbstverständnis und Selbstkontextualisierung untersucht. Flecks Verhältnis zur Soziologie ist dabei ebenso ambivalent wie komplex. Wohl votiert Fleck vehement für ein explizit soziologisches Programm - für eine Soziologie des Denkens, doch letztere schließt nur bedingt an die referenzierte soziologische Literatur und deren Autoren an. Vielmehr setzt sich Fleck kritisch ab. Eine Ausnahme bildet Georg Simmel mit seinem klassischen Werk Soziologie von 1908. Während Durkheim und seine Schule sowie Jerusalem und andere von Fleck aufgrund ihrer Auseinandersetzung mit kollektiven Grundlagen des Denkens zitiert und dann für ihre entwicklungssoziologischen Ansätze kritisiert werden, die das wissenschaftliche Denken aus kollektiver Befangenheit herauslösen, individualisieren, objektivieren, rationalisieren und abstrahieren wollen, wird Simmel für seine Theorie des sozialen Differenzierung und als Zeuge für die Anerkennung der „Wirkung des sozialen Verkehrs der Denkgebilde" in der Soziologie positiv - oder zumindest nicht negativ referenziert. Fleck teilt mit Simmel ein Interesse für die - wie es im Untertitel von Simmels Soziologie heißt - „Formen der Vergesellschaftung“, die immer auch kognitive, psychologische und kulturelle Aspekte umfassen, auch Denkund Wissenschaftsformen. Fleck übernimmt von Simmel verschiedene Konzepte zur formalen Beschreibung von Entstehung und Entwicklung von Denkkollektiven, und in der Tat ist die formale Analyse des Sozialen jenes genuine Feld, wofür die Soziologie nach Simmels Auffassung im Besonderen zuständig sein solle. Mit Simmel lassen sich daher implizite Gehalte bei Fleck explizieren und damit der Fleck'sche Standpunkt fruchtbar rekonstruieren. 
Umgekehrt gibt es bei Fleck viele Berührungspunkte zu den der Soziologie nahestehenden oder mit ihr im permanenten Austausch stehenden Disziplinen Psychologie, Ethnologie, Volkskunde und Geschichte. Im Schnittpunkt dieser Fächer stand auch die zu Flecks Zeiten bereits weitgehend vergessene Völkerpsychologie. Hier drängt sich einmal mehr der aus dieser Tradition stammende Simmel, der wie kein anderer eine Brücke von der Völkerpsychologie in die Soziologie baute, als explizite Referenz und reiche heuristische und systematische Quelle für eine historische und theoretische Rekonstruktion des Fleck'schen Ansatzes - jedenfalls aber als anschlussfähige Bereicherung in der Auseinandersetzung mit Fleck - auf. Solche Exploration muss letztlich genauso auf eine kulturwissenschaftliche wie soziologische Kontextualisierung Flecks zielen, oder - um eine von Klaus Christian Köhnke auf Simmel gemünzte Formel aufzunehmen - auf eine Annäherung an Fleck als Vertreter einer „Soziologie als Kulturwissenschaft“ (Köhnke 1990).

Eine solche Rekonstruktion von Flecks Wissenschaft des Sozialen sollte natürlich nicht die zentralen Anliegen und Stärken des Autors verdecken, sondern vertiefend bekräftigen. Sie zielen nicht - wie bei Simmel - auf eine allgemeine Gesellschafts- oder Kulturtheorie, sondern sind spezifisch auf die Erforschung von medizinischer beziehungsweise wissenschaftlicher Wissensproduktion gerichtet. Zu Recht wird Fleck als Pionier und Klassiker eines practical und cultural turn in der Wissenschaftsforschung gewürdigt, der auch sehr früh auf die materiellen Aspekte von Apparaten und anderen Objekten hingewiesen hat. Weiterhin inspirierend bleiben seine Konzeptualisierungen auch für Erforschung der Beziehungen von Experten und Laien (Löwy 2005). Der Umstand, dass er stets aus der Warte eines erfahrenen Mediziners und Mikrobiologen argumentierte, sättigt seine Analysen durch Fach- beziehungsweise Feldkompetenz. Ob Bruno Latour wohl dies im Auge hatte, als er behauptete, Fleck hätte mit vierzig Jahren Vorsprung ein Programm entwickelt, das dem Strong Programme von David Bloor und anderen zum Verwechseln ähnlich sähe (Latour 2005: 253)? Solcher Positionierung, die Fleck wieder sehr stark an die Soziologie der Wissenschaft rückbindet, stehen transgressivere Würdigungen gegenüber, die Flecks Verdienst herausstreichen, die „Wissenschaftstheorie aus dem engen Kreis der scientific community herauszulösen" und einen wissenschaftstheoretisch und - historisch gut umsetzbaren Ansatz zur Analyse „lokal besonderer" Wissenskulturen anzubieten (Werner/Zittel 2011: 15). Flecks Lehre vom Denkstil und Denkkollektiv bietet aber auch Hand, Vernetzungen und Interaktionen - Wechselwirkungen - lokaler Wissenskulturen konzeptuell zu integrieren. Mit Fleck lässt sich wissensorientierte Vergesellschaftung begreifen. Mit seiner Orientierung auf Prozesse und Interaktionen, die sich nicht zu einem Ganzen fügen müssen, offeriert Fleck damit auch einen Beitrag zu einer nicht-systemtheoretischen Sozialtheorie von Wissensgesellschaft. 


\section{Anmerkungen}

1 Kuhn rapportiert im Vorwort zu Structure of Scientific Revolutions über seine Arbeit an diesem Buch als Junior Fellow in Harvard, nebst der Bemerkung eines anderen Juniormitglieds, Francis X. Sutton, hätte Fleck ihn zur Einsicht gebracht, seine Gedanken müssten wohl in den Rahmen einer Soziologie der wissenschaftlichen Gemeinschaft gestellt werden. Für seine Aufnahme gestaltpsychologischer Ideen verwies Kuhn dagegen nicht auf Fleck, sondern auf einen nicht namentlich genannten Kollegen. Auch Flecks historische und philosophisch-epistemologische Seiten wurden von Kuhn nicht als Einfluss genannt (Kuhn [1962] 1976: 8)

2 Die expliziten soziologischen Bezüge im Fleck'schen Werk werden bei Egloff 2007b einer Würdigung unterzogen, welche auch die der Monographie vorausgehenden Aufsätze miteinbezieht, jedoch Flecks Referenz auf Georg Simmel nicht weiter vertieft.

3 Für eine ausführlichere Darstellung der Diskrepanz in der soziologischen Fachentwicklung zwischen den USA und Europa siehe Egloff 2007a, S. 1-15. Zur europäischen Verzögerung in der soziologischen Fachentwicklung siehe auch Wagner 1991.

4 Egloff 2007b. Auf die Ausnahme Georg Simmels als ausschließlich positive soziologische Referenz wird weiter unten zurückzukommen sein.

5 Klassische Beiträge zur Verortung Flecks zwischen Soziologie, Philosophie und Medizin wurden von Schnelle 1982 und Löwy 1990 vorgelegt. Griesecke 2008 hat für eine Rekonstruktion Flecks vergleichender Erkenntnistheorie zudem die Parallelen zu Strömungen in der Ethnographie des 20. Jahrhunderts herausgearbeitet.

6 Bezeichnenderweise wird Simmel auch in Baldamus' Einschätzung oben nicht genannt, obwohl ihn Fleck deutlich referenziert.

7 Für eine Rekontextualisierung Flecks im Rahmen der Entstehung einer historischen Epistemologie siehe Rheinberger 2006.

8 Eine jüngste Bestimmung Fleck'scher Positionierung mit analytisch-philosophischer und wissenssoziologischer Perspektive findet sich bei Seidel 2011.

9 Für einen Versuch, Fleck mit der Philosophie Hegels in Verbindung zu setzen, siehe Rosenhagen 2011.

10 Diese Beziehung muss allerding in vieler Hinsicht ambivalent beziehungsweise reserviert bleiben, nicht nur weil bei Fleck die Entwicklung und der Vergleich von Denkstilen im Vordergrund steht, während kaum ein Interesse am Konzept eines mehr oder weniger homogenen „Volkes“ vorliegt.

11 Wie weiter unten auszuführen ist, verweist Fleck mit Simmel jedoch explizit auf ein soziologisches Programm, das klar in der völkerpsychologischen Tradition steht.

12 Fleck verweist auf Jerusalem 1924, wo dieser wiederum (S. 192) auf seinen eigenen Aufsatz „Soziologie des Erkennens“ von 1909 referiert, in welchem er diesen Begriff zum ersten Mal dargelegt und erläutert habe.

13 Für eine Kritik dieser Kritik siehe jüngst Uebel 2012.

14 Auch Sauerland 2007 hebt die wichtige Rolle Simmels für Fleck hervor. Er nennt Simmel gar „ein großes Vorbild für Fleck“ (ebd.: 77).

15 Der Rest der außergewöhnlich detaillierten Fußnote befasst sich mit psychologischen Diskussionen zu Massen- beziehungsweise Kollektivwahrnehmungen (ebd.: 145).

\section{Literatur}

Baldamus, William, 1977. Ludwik Fleck and the Development of the Sociology of Science. In: Peter Gleichmann, Hg., Human Figurations. Essays for/Aufsätze für Norbert Elias. Amsterdam: Amsterdams Sociologisch Tijdschrift, 135-156.

Böhme, Hartmut/Matussek, Peter/Müller, Lothar, 2002. Orientierung Kulturwissenschaft, 2. Aufl. Reinbek: Rowohlt. 
Cohen, Robert S./Schnelle, Thomas, Hg., 1986. Cognition and Fact: Materials on Ludwik Fleck. Dordrecht: Reidel.

Egloff, Rainer, 2007a. Krise und Disziplin. Zur Entstehung der Fachsoziologie an der University of Chicago. Diss. Univ. Zürich.

Egloff, Rainer, 2007b. Leidenschaft und Beziehungsprobleme: Ludwik Fleck und die Soziologie. In: Bozena Choluj und Jan C. Joerden, Hg., Von der wissenschaftlichen Tatsache zur Wissensproduktion. Ludwik Fleck und seine Bedeutung für die Wissenschaft und Praxis. Frankfurt a. M.: Peter Lang, 79-93.

Fleck, Ludwik, [1935] 1979. Genesis and Development of a Scientific Fact. Hg. von Thaddeus J. Trenn und Robert K. Merton. Chicago: University of Chicago Press.

Fleck, Ludwik, [1935] 1980. Entstehung und Entwicklung einer wissenschaftlichen Tatsache. Einführung in die Lehre vom Denkstil und Denkkollektiv. Hg. von Lothar Schäfer und Thomas Schnelle. Frankfurt a. M.: Suhrkamp.

Fleck, Ludwik, 1983. Erfahrung und Tatsache. Gesammelte Aufsätze. Hg. von Lothar Schäfer und Thomas Schnelle. Frankfurt a. M.: Suhrkamp.

Graevenitz, Gerhart von, 1999. „Verdichtung“. Das Kulturmodell der Zeitschrift für Völkerpsychologie und Sprachwissenschaft. Kea, 12, 19-57.

Griesecke, Birgit, 2008. Vergleichende Erkenntnistheorie. Einführende Überlegungen zum Grundkonzept der Fleckschen Methodologie. In: dies. und Erich Otto Graf, Hg., Ludwik Flecks vergleichende Erkenntnistheorie. Die Debatte in Przeglad Filozoficny 1936-1937. Berlin: Parerga, 9-59.

Harwood, Jonathan, 1986. Ludwik Fleck and the Sociology of Knowledge, Review. Social Studies of Science, 16, 173-187.

Jerusalem, Wilhelm, 1924. Die soziologische Bedingtheit des Denkens und der Denkformen. In: Max Scheler, Hg., Versuche zu einer Soziologie des Wissens. München: Duncker \& Humblot, 182-202.

Klautke, Egbert, 2010. The Mind of the Nation: The Debate about Völkerpsychologie, 1851-1900. Central Europe, 8, 1-19.

Köhnke, Klaus Christian, 1990. Soziologie als Kulturwissenschaft: Georg Simmel und die Völkerpsychologie. Archiv für Kulturgeschichte, 72, 223-232.

Köhnke, Klaus Christian, 1996. Der junge Simmel - in Theoriebeziehungen und sozialen Bewegungen. Frankfurt a. M.: Suhrkamp.

Köhnke, Klaus Christian 2003. Einleitung. In: Moritz Lazarus, Grundzüge der Völkerpsychologie und Kulturwissenschaft. Hamburg: Meiner, IX-XLII, IX.

Kuhn, Thomas S. [1962] 1976. Die Struktur wissenschaftlicher Revolutionen. 2. Aufl. Frankfurt a. M.: Suhrkamp.

Latour, Bruno 2005. Transmettre la syphilis. Partager l'objecitivité. In: Ludwik Fleck, Genèse et développement d'un fait scientifique. Paris: Les Belles Lettres, 251-260.

Lazarus, Moritz [1851] 2003. Ueber den Begriff und die Möglichkeit einer Völkerpsychologie. In: ders., Grundzüge der Völkerpsychologie und Kulturwissenschaft. Hg. von Klaus Christian Köhnke, Hamburg: Meiner, 3-25.

Lazarus, Moritz [1862] 2003. Verdichtung des Denkens in der Geschichte. Ein Fragment. In: ders., Grundzüge der Völkerpsychologie und Kulturwissenschaft. Hg. von Klaus Christian Köhnke, Hamburg: Meiner, 27-38.

Lazarus, Moritz [1883/1862] 2003. Ueber das Verhältniß des Einzelnen zur Gesammtheit. In: ders., Grundzüge der Völkerpsychologie und Kulturwissenschaft. Hg. von Klaus Christian Köhnke, Hamburg: Meiner, 39-129.

Löwy, Ilana, 1990. The Polish School of Philosophy and Medicine. From Tytus Chalubinski (18201889) to Ludwik Fleck (1896-1961). Dordrecht: Kluwer.

Löwy, Ilana, 2005. Fleck dans son temps, Fleck dans notre temps. Genèse et développement d'une pensée, Préface. In: Ludwik Fleck, Genèse et développement d'un fait scientifique. Paris: Les Belles Lettres, IX-XLII.

Nedelmann, Brigitta, 1995. Georg Simmel als Klassiker soziologischer Prozeßanalysen. In: HeinzJürgen Dahme und Otthein Rammstedt, Hg., Georg Simmel und die Moderne. Neue Interpretationen und Materialien. 2. Aufl. Frankfurt a. M.: Suhrkamp, 91-115.

Rheinberger, Hans-Jörg, 2006. Zur Historizität wissenschaftlichen Wissens. Ludwik Fleck, Edmund Husserl. In: ders., Epistemologie des Konkreten. Studien zur Geschichte der modernen Biologie. Frankfurt a. M.: Suhrkamp, 21-36. 
Rosenhagen, Raja, 2011. Fleck und Hegel über Wahrheit als Station in einer Entwicklung. In: Rainer Egloff und Johannnes Fehr, Hg., Vérité, Widerstand, Development: At Work with/ Arbeiten mit/Travailler avec Fleck. Zürich: Collegium Helveticum, 157-169.

Sauerland, Karol, 2007. Ludwik Flecks unerwünschter soziologischer Blick. In: Bozena Choluj und Jan C. Joerden, Hg., Von der wissenschaftlichen Tatsache zur Wissensproduktion. Ludwik Fleck und seine Bedeutung für die Wissenschaft und Praxis. Frankfurt a. M.: Peter Lang, 6577.

Schnelle, Thomas, 1982. Ludwik Fleck - Leben und Denken. Zur Entstehung und Entwicklung des soziologischen Denkstils in der Wissenschaftsphilosophie. Freiburg: Hochschulverlag.

Schnelle, Thomas, 1986. Microbiology and Philosophy of Science, Lwow and the German Holocaust: Stations of a Life - Ludwik Fleck 1896-1961. In: Robert S. Cohen and Thomas Schnelle, Hg., 1986. Cognition and Fact: Materials on Ludwik Fleck. Dordrecht: Reidel, 3-36.

Seidel, Markus, 2011. Relativism or Relationism? A Mannheimian Interpretation of Fleck's Claims About Relativism. Journal for General Philosophy of Science, 42, 219-240.

Simmel, Georg [1908] 1992. Soziologie. Untersuchungen über die Formen der Vergesellschaftung. Frankfurt a. M.: Suhrkamp.

Tyrell, Hartmann/Rammstedt, Otthein/Meyer, Ingo, Hg., 2011. Georg Simmels große „Soziologie“. Eine kritische Sichtung nach hundert Jahren. Bielefeld: Transcript.

Uebel, Thomas, 2012. But is it Sociology of Knowledge? Wilhelm Jerusalem's „Sociology of Cognition" in Context. Studies in Eastern European Thought, 64, 5-37.

Wagner, Peter, 1991. Science of Society Lost: On the Failure to Establish Sociology in Europe during the ,, Classical“ Period. In: Richard P. Whitley, Björn Wittrock und Peter Wagner, Hg., Dicourses on Society. The Shaping of the Social Science Disciplines. Dordrecht: Kluwer, 219245.

Werner, Sylwia/Zittel, Claus, 2011. Einleitung: Denkstile und Tasachen, In: dies., Hg., 2011. Ludwig Fleck. Denkstile und Tatsachen. Gesammelte Schriften und Zeugnisse. Berlin: Suhrkamp, 9-38.

Rainer Egloff

Collegium Helveticum

Schmelzbergstrasse 25

8092 Zürich

Schweiz

E-Mail: Egloff@collegium.ethz.ch 